conserved in mature animals and human disease states, clear demonstration of the validity of such predictions is often lacking and requires complex genetic and physiologic approaches. The studies in this issue of the JCI identify AM signaling as a key regulator of blood and lymphatic vessel function, but whether Starling would be disappointed or gratified to learn of the persistent significance of his experiments in our molecular and genetic age is not clear. Clear definition of the vascular roles of AM signaling will parallel progress in addressing these broad issues in vascular biology, leaving plenty of future work for these and other investigators.

\section{Acknowledgments}

I thank the members of my laboratory and Guillermo Oliver for interesting discussions.

Address correspondence to: Mark L. Kahn, Department of Medicine, University of Pennsylvania, 952 BRB II/III, 421 Curie Blvd., Philadelphia, Pennsylvania 19104, USA. Phone: (215) 898-9007; Fax: (215) 573-2094; E-mail: markkahn@mail. med.upenn.edu.
1. Adams, R.H., and Alitalo, K. 2007. Molecular regulation of angiogenesis and lymphangiogenesis. Nat. Rev. Mol. Cell Biol. 8:464-478.

2. Sabin, F. 1901. On the origin of the lymphatic system from the veins and the development of the lymph hearts and thoracic duct in the pig. Am.J. Anat. 4:367-389.

3. Srinivasan, R.S., et al. 2007. Lineage tracing demonstrates the venous origin of the mammalian lymphatic vasculature. Genes Dev. 21:2422-2432.

4. Wang, H.W., et al. 2004. Kaposi sarcoma herpesvirus-induced cellular reprogramming contributes to the lymphatic endothelial gene expression in Kaposi sarcoma. Nat. Genet. 36:687-693.

5. Hong, Y.K., et al. 2004. Lymphatic reprogramming of blood vascular endothelium by Kaposi sarcomaassociated herpesvirus. Nat. Genet. 36:683-685.

6. Fritz-Six, K.L., Dunworth, W.P., Li, M., and Caron, K.M. 2008. Adrenomedullin signaling is necessary for murine lymphatic vascular development. J. Clin. Invest. 118:40-50.

7. Ichikawa-Shindo, Y., et al. 2008. The GPCR modulator protein RAMP2 is essential for angiogenesis and vascular integrity. J. Clin. Invest. 118:29-39.

8. Kitamura, K., et al. 1993. Adrenomedullin: a novel hypotensive peptide isolated from human pheochromocytoma. Biochem. Biophys. Res. Commun. 192:553-560.

9. Gibbons, C., Dackor, R., Dunworth, W., Fritz-Six, K., and Caron, K.M. 2007. Receptor activity-modifying proteins: RAMPing up adrenomedullin signaling. Mol. Endocrinol. 21:783-796.

10. Czyzyk, T.A., et al. 2005. Deletion of peptide amidation enzymatic activity leads to edema and embryonic lethality in the mouse. Dev. Biol. 287:301-313.

11. McLatchie, L.M., et al. 1998. RAMPs regulate the transport and ligand specificity of the calcitoninreceptor-like receptor. Nature. 393:333-339.
12. Caron, K.M., and Smithies, O. 2001. Extreme hydrops fetalis and cardiovascular abnormalities in mice lacking a functional Adrenomedullin gene. Proc. Natl. Acad. Sci. U. S. A. 98:615-619.

13. Shindo, T., et al. 2001. Vascular abnormalities and elevated blood pressure in mice lacking adrenomedullin gene. Circulation. 104:1964-1971.

14. Starling, E.H. 1896. On the absorption of fluids from connective tissue spaces. J. Physiol. Lond. 19:312-326.

15. Michel, C.C. 1997. Starling: the formulation of his hypothesis of microvascular fluid exchange and its significance after 100 years. Exp. Physiol. 82:1-30.

16. Karkkainen, M.J., et al. 2004. Vascular endothelial growth factor $\mathrm{C}$ is required for sprouting of the first lymphatic vessels from embryonic veins. Nat. Immunol. 5:74-80.

17. Machin, G.A. 1989. Hydrops revisited: literature review of 1,414 cases published in the 1980 s. Am. J. Med. Genet. 34:366-390.

18. Dackor, R.T., et al. 2006. Hydrops fetalis, cardiovascular defects, and embryonic lethality in mice lacking the calcitonin receptor-like receptor gene. Mol. Cell. Biol. 26:2511-2518.

19. Gale, N., et al. 2002. Angiopoietin-2 is required for postnatal angiogenesis and lymphatic patterning, and only the latter role is rescued by angiopoietin-1. Dev. Cell. 3:411.

20. Makinen, T., et al. 2005. PDZ interaction site in ephrinB2 is required for the remodeling of lymphatic vasculature. Genes Dev. 19:397-410.

21. Wigle, J.T., and Oliver, G. 1999. Prox1 function is required for the development of the murine lymphatic system. Cell. 98:769-778.

22. Banerji, S., et al. 1999. LYVE-1, a new homologue of the CD44 glycoprotein, is a lymph-specific receptor for hyaluronan. J. Cell Biol. 144:789-801.

\title{
Anticonvulsant effects of leptin in epilepsy
}

\author{
Sabrina Diano1,2 and Tamas L. Horvath ${ }^{1,2,3}$ \\ 1Department of Obstetrics, Gynecology \& Reproductive Sciences, ${ }^{2}$ Department of Neurobiology, and \\ ${ }^{3}$ Section of Comparative Medicine, Yale School of Medicine, New Haven, Connecticut, USA.
}

\begin{abstract}
Secreted from adipose tissue at levels proportional to fat stores, the hormone leptin is a critical regulator of the hypothalamic machinery that controls feeding and energy metabolism. Despite the critical role of leptin in the maintenance of energy homeostasis, no leptin-based therapeutic approaches have emerged to combat metabolic disorders such as obesity or diabetes. In this issue of the JCI, Xu et al. report a robust influence of leptin, beyond its role in metabolism, on hippocampal neuronal processes implicated in the etiology of epileptic seizures, learning, and memory (see the related article beginning on page 272). They show, in two rodent seizure models, that leptin administered directly to the brain or nasal epithelium suppresses seizures via direct effects on glutamate neurotransmission in the hippocampus. These observations suggest that leptin may have therapeutic potential in the treatment of epilepsy and strengthen the notion that peripheral metabolic hormones such as leptin play important roles in the regulation of higher brain functions.
\end{abstract}

Nonstandard abbreviations used: AMPA, $\alpha$-amino-3hydroxy-5-methyl-4-isoxazole proprionic acid.

Conflict of interest: The authors have declared that no conflict of interest exists.

Citation for this article: $J$. Clin. Invest. 118:26-28 (2008). doi:10.1172/JCI34511.
Over the last century, it has been hypothesized and then experimentally proven that a humoral signal from the periphery, specifically from adipose tissue, plays a role in the regulation of feeding and energy metabolism (1-5). The identity of this hormone, named leptin, was elegantly revealed by Friedman and colleagues in 1994 (6), and thus began a new era in the use of molecular biology in the quest to identify a cure for metabolic disorders, most notably obesity. This discovery revolutionized the field and attracted increasing numbers of creative researchers with sophisticated tools in an effort to determine the mechanisms of action of leptin in the hypothalamus and its role in the regulation of feeding and adiposity (reviewed in ref. 7). To date, despite the great wealth of knowledge that has been gained and the discovery of new hormones, no leptin-based medical remedy has emerged for the treatment of obesity. While new avenues of research in this area are being pursued by many, the study by $\mathrm{Xu}$ et al. reported in this issue of the JCI (8) reveals that the leptin signaling pathway has important functions that go beyond 


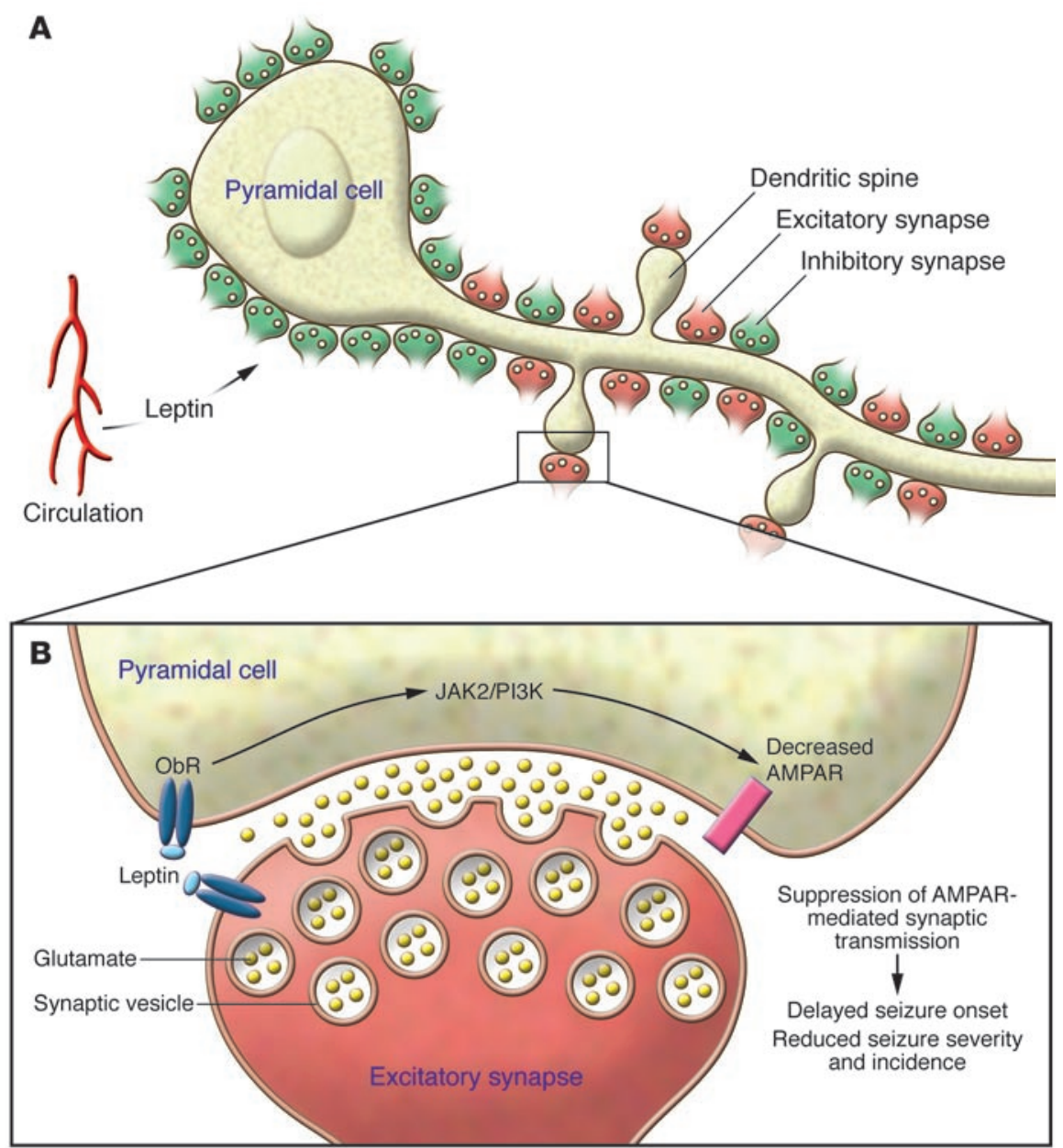

its role in the regulation of food intake and metabolism. In their study, these authors describe the mechanisms of action of leptin in the hippocampus and show that leptin opposes the propagation of seizures in two rodent seizure models. They demonstrate that this effect can be achieved by nasal administration of the hormone, thereby strengthening the potential for the use of leptin in anticonvulsant therapies.

\section{Current epilepsy therapeutics}

The cause of epilepsy varies among patients and involves various brain structures. Temporal lobe epilepsy is related to malfunctioning of excitatory neurotransmission of the hippocampal formation. While surgery may be an option when the brain region responsible for seizure generation has been identified and is responsible for no vital functions, the most commonly used therapeutic approach to control seizures is pharmacological. The majority of the pharmaceutical compounds available to treat epilepsy target neurotransmitter systems in an effort to slow excitatory, glutamate transmission either by directly antagonizing glutamate receptors or enhancing the tone of the inhibitory neurotransmitter GABA (9). Because glutamate and GABA are ubiquitous neurotransmitters throughout the CNS, the side effects of these therapies involve a broad array of brain mechanisms, from the regulation of homeostasis to alterations in higher brain functions. Thus, new candidate epilepsy therapeutics would ideally selectively target molecules with restricted distribution in the brain, which may reduce side effects that have an impact on other brain functions. Leptin receptors are not ubiquitous in the brain; however, they are expressed in the hippocampal formation (10).

\section{Action of leptin on seizures}

Xu et al. (8) tested leptin's anticonvulsant action in two rodent seizure models either by directly injecting leptin into the cortex or by intranasal administration (Figure 1). They found that focal seizures induced by

\section{Figure 1}

Schematic illustration of the anticonvulsant effect of blood-borne leptin on hippocampal principal cells. (A) Leptin derived from the circulation targets pyramidal cells of the hippocampal formation that are innervated by excitatory (red) and inhibitory (green) synapses. Excitatory synapses are frequently established on dendritic spines of pyramidal cells. (B) The report by $\mathrm{Xu}$ et al. (8) in this issue of the $\mathrm{JCl}$ shows that intranasal leptin administration in two rodent seizure models has an anticonvulsant effect. The effect of leptin on synaptic transmission in the hippocampus involves leptin binding to its receptor, followed by activation of JAK2/PI3K signaling pathways induced by the long form of the leptic receptor $(\mathrm{ObR})$, which alters both pre- and postsynaptic mechanisms and leads to suppression of glutamate-induced excitatory postsynaptic events mediated by AMPA-type glutamate receptors (AMPAR). This suppressed excitatory neurotransmission explains leptin's anticonvulsant effects. neocortical injections of 4-aminopyridine (4AP) in rats were shortened and reduced in number by coinjection with leptin. In addition, leptin reduced neuronal spiking in an in vitro seizure model. In mice, intranasal administration of leptin produced elevated brain and serum leptin levels and delayed the onset of pentylenetetrazole-induced generalized convulsive seizures. In identifying the underlying mechanism, leptin was found to inhibit ionotropic $\alpha$-amino3-hydroxy-5-methyl-4-isoxazole proprionic acid (AMPA) glutamate receptor-mediated synaptic transmission in hippocampal slices (Figure 1B). This inhibition was found to be leptin-receptor dependent, as leptin failed to inhibit synaptic responses in slices from leptin receptor-deficient $d b / d b$ mice. This study reveals that, in agreement with previous findings on leptin's effect on the hippocampus and seizures (11), it is the JAK2/PI3K pathway (leptin is known to signal through its receptor by activating JAK/STAT and JAK2/PI3K pathways) that is likely to be involved, as $\mathrm{Xu}$ et al. show 
that JAK2 and PI3K antagonists prevented leptin inhibition of AMPA receptor-regulated synaptic transmission (Figure 1). In light of the results of Xu et al. (8) and earlier observations that leptin levels may be increased during consumption of a ketogenic diet (12), it is plausible that leptintriggered signaling may play a role in the known beneficial effects of a ketogenic diet on suppression of seizures (13).

\section{The hippocampus is more than a seizure-controlling device}

The elaboration of the molecular mechanism through which leptin alters AMPA receptor activity is an important addition to our understanding of how leptin can affect hippocampal synaptic transmission during physiological and pathological conditions. However, there are many questions that need to be addressed before a true sense of the usefulness of leptin can be assessed regarding therapies for epilepsy. First, the rodent models tested by Xu et al. (8) are acute models of seizure generation, and seizures were generated in the face of elevated leptin levels either via cortical or nasal application of the hormone. In almost all cases of epilepsy, patients are identified and treated after the first episode of a seizure has occurred. Thus, it will be important to determine whether, in chronic seizure models, post hoc administration of leptin specifically via the nasal route will have beneficial effects. An equally intriguing aspect of leptin action is its inability to affect brain functions associated with food intake and energy expenditure in diet-induced obese animals. This so-called leptin resistance, may occur due to changes in blood-brain barrier function (reviewed in ref. 14) and/or intraneuronal events (15), and it will be important to determine whether this resistance also emerges in the hippocampus. This is specifically significant, as the human population, most alarmingly children, is becoming more overweight and moving toward an increased likelihood of leptin resistance. The data acquired and knowledge gained from the utilization of available animal models will be vital in order to determine the viability of potential therapies.

Suppression of glutamate transmission by leptin can diminish the likelihood of seizure generation and propagation. The flip side of the coin is that this same glutamate-regulated synaptic transmission in the hippocampus is critical for spatial learning and memory. If leptin were to be considered as a putative epilepsy therapy, an intriguing option would be to determine to what extent the suppression of hippocampal synaptic events by leptin might interfere with learning and memory. Furthermore, as mentioned above, the US and most Western societies are experiencing a trend of increasing body mass index, which is associated with increasing circulating leptin levels. Whether increasing levels of leptin in the circulation would have an overall negative impact on higher brain functions or would be irrelevant due to leptin resistance are important questions to entertain in light of recent studies showing the U-shaped nature of the doseresponse curve of leptin in hippocampal function, in which high doses of leptin impair spatial learning and memory performance $(16,17)$.

Undoubtedly, the potential of leptin to suppress initiation and propagation of seizures could fill a critical medical need. Clinically, the most significant message of the study by Xu et al. (8) is the hope for the possibility of interfering with seizure initiation and propagation by leptin via an intranasal route of application. Should the approach be feasible and effective in humans, a new therapy could rapidly be on the way. Equally intriguing is the trend of moving the focus on therapeutic potentials for metabolic hormones, such as leptin and ghrelin, from classical metabolic disorders, such as obesity and diabetes, to higher brain functions (18) and neurodegenerative disorders of the brain, including epilepsy. In light of the emerging recognition of the critical role of impaired cell metabolism in most of these disorders (19), the future may hold greater promise for therapeutic success using metabolic hormones and their signaling modalities to combat neurodegenerative disorders rather than obesity.

Address correspondence to: Tamas L. Horvath or Sabrina Diano, Section of Comparative Medicine and Departments of Ob/Gyn, and Neurobiology, Yale University, 375 Congress Avenue, LSOG 117, PO Box 208016, New Haven, Connecticut 06510-
8016, USA. Phone: (203) 785-2525; Fax: (203) 785-7499. E-mail: tamas.horvath@ yale.edu (T.L. Horvath); sabrina.diano@ yale.edu (S. Diano).

1. Coleman, D.L., and Hummel, K.P. 1969. Effects of parabiosis of normal with genetically diabetic mice. Am. J. Physiol. 217:1298-1304.

2. Coleman, D.L. 1973. Effects of parabiosis of obese with diabetes and normal mice. Diabetologia. 9:294-298.

3. Campfield, L.A., Smith, F.J., Guisez, Y., Devos, R., and Burn, P. 1995. Recombinant mouse OB protein: evidence for a peripheral signal linking adiposity and central neural networks. Science. 269:546-549.

4. Halaas, J.L., et al. 1995. Weight-reducing effects of the plasma protein encoded by the obese gene. Science. 269:543-546.

5. Pelleymounter, M.A., et al. 1995. Effects of the obese gene product on body weight regulation in ob/ob mice. Science. 269:540-543.

6. Zhang, Y., et al. 1994. Positional cloning of the mouse obese gene and its human homologue. Nature. 372:425-432.

7. Gao, Q., and Horvath, T.L. 2007. Neurobiology of feeding and energy expenditure. Annu. Rev. Neurosci. 30:367-398.

8. Xu, L., et al. 2008. Leptin inhibits 4-aminopyridine- and pentylenetetrazole-induced seizures and AMPAR-mediated synaptic transmission in rodents. J. Clin. Invest. 118:272-280.

9. Schachter, S.C. 2007. Currently available antiepileptic drugs. Neurotherapeutics. 4:4-11.

10. Mercer, J.G., et al. 1996. Localization of leptin receptor mRNA and the long form splice variant (Ob-Rb) in mouse hypothalamus and adjacent brain regions by in situ hybridization. FEBS Lett. 387:113-116.

11. Shanley, L.J., O'Malley, D., Irving, A.J., Ashford, M.L., and Harvey, J. 2002. Leptin inhibits epileptiform-like activity in rat hippocampal neurones via PI 3-kinase-driven activation of BK channels. J. Physiol. 545:933-944.

12. Thio, L.L., Erbayat-Altay, E., Rensing, N., and Yamada, K.A. 2006. Leptin contributes to slower weight gain in juvenile rodents on a ketogenic diet. Pediatr. Res. 60:413-417.

13. Murphy, P. 2005. Use of the ketogenic diet as a treatment for epilepsy refractory to drug treatment. Expert Rev. Neurother. 5:769-775.

14. Banks, W.A. 2006. The blood-brain barrier as a regulatory interface in the gut-brain axes. Physiol. Behav. 89:472-476.

15. Bjørbaek, C., Elmquist, J.K., Frantz, J.D., Shoelson, S.E., and Flier, J.S. 1998. Identification of SOCS-3 as a potential mediator of central leptin resistance. Mol. Cell. 1:619-625.

16. Oomura, Y., et al. 2006. Leptin facilitates learning and memory performance and enhances hippocampal CA1 long-term potentiation and CaMK II phosphorylation in rats. Peptides. 27:2738-2749.

17. Farr, S.A., Banks, W.A., and Morley, J.E. 2006. Effects of leptin on memory processing. Peptides. 27:1420-1425.

18. Diano, S., et al. 2006. Ghrelin controls hippocampal spine synapse density and memory performance. Nat. Neurosci. 9:381-388.

19. Andrews, Z.B., Diano, S., and Horvath, T.L. 2005. Mitochondrial uncoupling proteins in the CNS: in support of function and survival. Nat. Rev. Neurosci. 6:829-840. 